\title{
TRIM14 promotes the migration and invasion of gastric cancer by regulating epithelial-to-mesenchymal transition via activation of AKT signaling regulated by miR-195-5p
}

\author{
FEIQIAN WANG, LITAO RUAN, JINRU YANG, QIAOLING ZHAO and WEI WEI \\ Department of Ultrasound Medicine, The First Affiliated Hospital of Xi'an Jiaotong University, \\ Xi'an, Shaanxi 710061, P.R. China
}

Received May 7, 2018; Accepted September 21, 2018

DOI: $10.3892 /$ or.2018.6750

\begin{abstract}
Tripartite motif-containing 14 (TRIM14) is a member of the TRIM protein family which has been implicated in several critical processes and is dysregulated in human cancers in a cancer-specific trend. However, its expression and function in human gastric cancer (GC) are still largely unknown. In this study, we confirmed for the first time that TRIM14 mRNA and protein were upregulated in GC tissues and cell lines as determined by qRT-PCR and western blot analysis. Clinical data disclosed that high TRIM14 expression was significantly associated with aggressive prognostic features, including advanced TNM stage and lymph node metastasis. In regards to 5-year survival, TRIM14 served as a potential prognostic marker for GC. Notably, TRIM14 promoted migration, invasion as measured by Transwell and epithelial-to-mesenchymal transition (EMT) as determined by western blot analysis and immunofluorescence (IF) in vitro and in vivo. Moreover, TRIM14 induced protein kinase B (AKT) pathway activation, and inhibition of AKT reversed the TRIM14-induced promotive effects on cell migration, invasion and EMT progression. Furthermore, we demonstrated that TRIM14 expression was regulated by miR-195-5p. miR-195-5p exerted an inhibitory role in GC migration and invasion. Finally, we confirmed that alteration of TRIM14 expression abolished the effects of miR-195-5p on GC cells. Conclusively, our results demonstrated that TRIM14 functions as an oncogene in regulating EMT and metastasis of GC via activating AKT signaling, which was regulated by miR-195-5p, supporting its potential utility as a therapeutic target for GC.
\end{abstract}

Correspondence to: Dr Litao Ruan, Department of Ultrasound Medicine, The First Affiliated Hospital of Xi'an Jiaotong University, 277 Yanta West Road, Xi'an, Shaanxi 710061, P.R. China

E-mail: ruanlt0502@163.com

Key words: TRIM14, gastric cancer, miR-195-5p, migration, EMT

\section{Introduction}

Gastric cancer (GC) is one of the most common malignancies and a leading cause of cancer-related deaths worldwide $(1,2)$. Despite the great improvements achieved in the diagnosis and treatment of gastric cancer, the long-term prognosis of these patients remains poor due to the high rate of invasion and metastasis (3). However, the underlying mechanisms remain largely unknown $(4,5)$. Therefore, there is an urgent need to unveil the molecular mechanisms underlying metastasis and identify novel therapeutic markers and develop new efficient treatment strategies.

Tripartite motif-containing 14 (TRIM14), which is located on chromosome $9 \mathrm{q} 22$, has been recognized as a cancer-related protein in previous studies (6-8). TRIM14 was found to be significantly upregulated in hepatocellular carcinoma and its high expression was demonstrated to be associated with poor prognosis of patients (9). Overexpression of TRIM14 was found to promote tongue squamous cell carcinoma aggressiveness by activating the $\mathrm{NF}-\kappa \mathrm{B}$ signaling pathway (10). In breast cancer, TRIM14 promoted cell proliferation and inhibited apoptosis via signal transducer and activator of transcription 3 (STAT3) signaling (11). These results suggest that TRIM14 is an oncogene in tumors. However, in non-small cell lung carcinoma (NSCLC), TRIM14 knockdown significantly enhanced tumor growth in NSCLC xenograft mouse models, while exogenous TRIM14 attenuated tumorigenesis. Moreover, the TRIM14 mRNA levels are significantly associated with poorer prognosis in early stage NSCLC patients (12). These results revealed that TRIM14 is a tumor suppressor. Therefore, the role of TRIM14 in tumors is tissue-specific. However, the expression levels and biological functions of TRIM14 in the progression of GC remain largely unknown.

Epithelial-to-mesenchymal transition (EMT) refers to the conversion of epithelial cells into cells with mesenchymal properties and appearance. EMT has been found to be a critical process during development and carcinogenesis (13). EMT has been validated to promote cancer cell dissemination and metastasis and maintain cancer stem cell properties in a variety of cancers $(14,15)$. Recently, EMT was found to confer high migration and invasion capacities, chemo-resistance, and 
strengthen the propensity for cancer metastasis (16). However, the regulatory mechanism of EMT in GC progression has not been entirely understood.

In the present study, we demonstrated that TRIM14 expression was significantly increased in human clinical GC tissues and cell lines. Its overexpression was associated with poor clinical features and poor survival. Gain- and loss-of-function experiments confirmed that TRIM14 regulated the migration and invasion of GC cells by alteration of the expression of EMT-associated factors. Moreover, we identified that the the protein kinase B (AKT) signaling pathway mediated the biological effects of TRIM14 in downstream and the upstream of TRIM expression was regulated by miR-195-5p in GC cells. Taken together, our findings suggest that TRIM14 plays a critical oncogenic role in GC progression and highlight its potential as a therapeutic target for GC treatment.

\section{Materials and methods}

Clinical samples and cell lines. A total of 117 GC tissues and matched adjacent non-tumor tissues were collected at at The First Affiliated Hospital of Xi'an Jiaotong University (Xi'an, China) from January 2007 to December 2009. Patients received no other curative strategy with chemotherapy or radiotherapy before surgery. The Ethical Committee of The First Affiliated Hospital of Xi'an Jiaotong University approved the study protocol and written informed consent was provided by all participating patients.

The human GC cell lines (MKN45, MGC803, BGC823, SGC7901 and AGS) and normal gastric epithelial cell line GES-1 which were obtained from the Chinese Academy of Sciences (Shanghai, China) were maintained in Invitrogen ${ }^{\mathrm{TM}}$ RPMI-1640 medium (Thermo Fisher Scientific, Inc., Waltham, MA, USA) added with $10 \%$ HyClone $^{\mathrm{TM}}$ fetal bovine serum (FBS; GE Healthcare Life Sciences, Logan, UT, USA) and $1 \%$ penicillin/streptomycin (HyClone; GE Healthcare Life Sciences). All cell lines were incubated in a humidified atmosphere with $5 \% \mathrm{CO}_{2}$ at $37^{\circ} \mathrm{C}$.

Cell transfection. Lipofectamine 2000 reagent (Invitrogen Life Technologies; Thermo Fisher Scientific, Inc.) was applied to conduct cells transfection on the basis of the product specification at a final concentration of $100 \mathrm{pmol}$. Mimic control (miR-control; CmiR0001-MR04), miR-195-5p mimics (HmiR0270), inhibitor control (anti-miR-NC; CmiR-AN0001-AM02) and miR-195-5p inhibitors (HmiR-AN0282) were purchased from Genecopoeia Inc. (Guangzhou, China). TRIM14 clones and TRIM14 shRNAs were obtained from Shanghai Sangon Biotech Co., Ltd. (Shanghai, China). Cells were transfected with the shRNAs and clones above using Lipofectamine 2000 according to the manufacturer's instructions. After $48 \mathrm{~h}$ of transfection, cells were used for the following experiments.

RNA extraction and quantitative real-time PCR ( $q R T-P C R)$. The total RNA from GC cells and tissues was extracted using Invitrogen $^{\mathrm{TM}}$ TRIzol reagent (Thermo Fisher Scientific, Inc.) according to the manufacturer's protocol.cDNA was synthesized by TaqMan miRNA reverse transcription (Applied Biosystems, Foster City, CA, USA) and a PrimeScript Reverse Transcriptase kit (Takara Biotechnology Co., Ltd., Dalian, China). The relative expression of miR-195-5p and TRIM14 mRNA were quantified using miRNA-specific TaqMan miRNA Assay Kit (Applied Biosystems) and the SYBR Premix Ex Taq ${ }^{\mathrm{TM}}$ Kit (Takara Bio Inc., Shiga, Japan) in the Applied Biosystems 7500 Sequence Detection system. The cycling conditions were as follows: First $95^{\circ} \mathrm{C}$ for $10 \mathrm{~min}$, then 40 cycles at $95^{\circ} \mathrm{C}$ for $15 \mathrm{sec}$ and $60^{\circ} \mathrm{C}$ for $60 \mathrm{sec}$. Primers for miR-195-5p and TRIM14 were obtained from Genecopoeia. The gene expression levels were calculated using the delta-delta Cq method with U6 or GAPDH as an internal control (17).

Immunofluorescence (IF). We used 4\% paraformaldehyde to fix transfected cells and used $0.3 \%$ Triton X-100 to permeabilize. The primary antibody E-cadherin, $\mathrm{N}$-cadherin and vimentin (dilution 1:300; Cell Signaling Technology, Danvers, MA, USA) was used. Then the Alexa Fluor-conjugated secondary antibody was performed in the next experiment. Lastly, the images were obtained by microscopy (Carl Zeiss, Oberkochen, Germany).

Western blotting. The whole proteins were lysed in RIPA buffer (Bio-Rad Laboratories, Hercules, CA, USA) supplemented with protease and phosphatase inhibitors (Roche) and the concentrations were quantified with BCA Protein Assay kit (Tiangen Biotech Co., Ltd., Beijing, China), and then $30 \mu \mathrm{g}$ protein was separated by $10 \%$ SDS-PAGE and transferred to PVDF membranes. Subsequently, the PVDF membranes were probed with primary antibodies against TRIM14 (dilution 1:1,000; cat. no. ab85374; Abcam, Cambridge, MA, USA), p-AKT (dilution 1:1,000; cat. no. 4060; Ser473; Cell Signaling Technology, Beverly, MA, USA), AKT (dilution 1:1,000; cat. no. 4691; Cell Signaling Technology), E-cadherin (dilution 1:1,000; cat. no. 14472; Cell Signaling Technology), vimentin (dilution 1:1,000; cat. no. 5741; Cell Signaling Technology), N-cadherin (dilution 1:1,000; cat. no. 14215; Cell Signaling Technology) and GAPDH (dilution 1:3,000; cat. no. sc-47724; Santa Cruz Biotechnology, Santa Cruz, CA, USA), and then probed with horseradish peroxidase (HRP)-conjugated secondary antibodies (dilution 1:5,000; cat. nos. 7074 and 7076; Cell Signaling Technology). The western blot was detected with enhanced chemiluminescence regent (Thermo Scientific Inc., Waltham, MA, USA) and analyzed using the Quantity One 1-D analysis software (Bio-Rad Laboratories, Inc.).

Luciferase reporter assay. The sequence of TRIM14 3'-untranslated region (UTR) containing the putative miR-195-5p binding region was amplified from human genomic DNA. Then the sequence was cloned into pGL3 luciferase reporter vector (Promega, Madison, WI, USA). The potential miR-195-5p binding sites were mutated by the Quick-change site-directed mutagenesis kit (Agilent Technologies, Santa Clara, CA, USA). The wild-type (wt) TRIM14 3'-UTR vector or mutant (mt) TRIM14 3'-UTR vector and miR-195-5p mimics or miR-195-5p inhibitors were co-transfected into MKN45 cells using Invitrogen ${ }^{\mathrm{TM}}$ Lipofectamine 2000 (Thermo Fisher Scientific, Inc.). The luciferase activity was measured using Dual-Luciferase Reporter Assay system (Promega) under a luminometer(Berthold Detection System,Pforzheim,Germany) and luciferase activity was normalized to Renilla activity. 
A

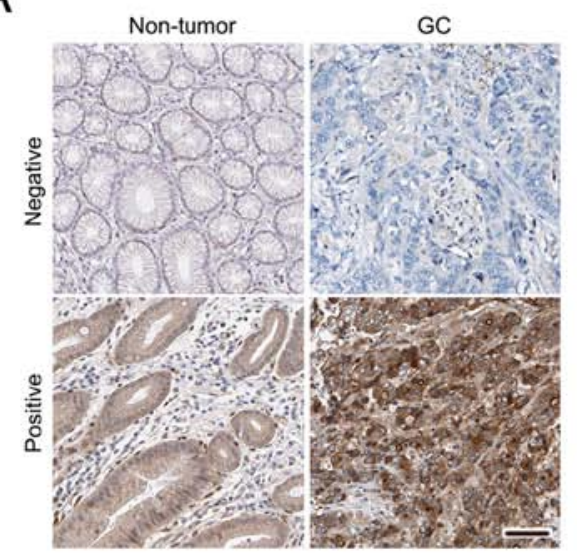

B

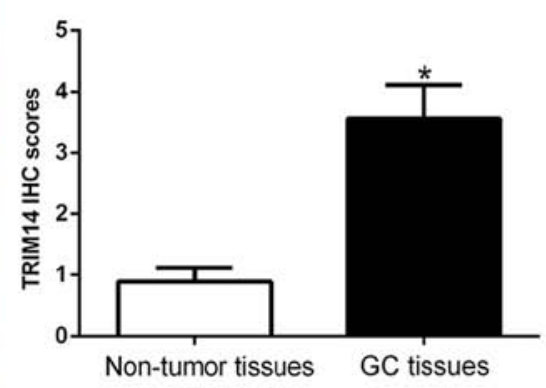

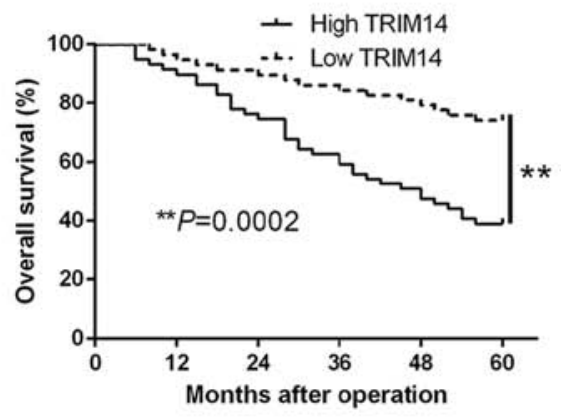

Figure 1. Expression of TRIM14 is correlated with the survival of GC patients. (A) Representative images of IHC staining of TRIM14 in differentiated GC and adjacent non-tumor tissues. Comparison of TRIM14 expression in HCC tissues was analyzed. (B) GC patients with higher expression of TRIM14 exhibited reduced overall survival. ${ }^{*} \mathrm{P}<0.05,{ }^{* *} \mathrm{P}<0.01$. TRIM14, tripartite motif-containing 14 ; GC, gastric cancer; IHC, immunohistochemistry.

Cell migration and invasion assays. The indicated GC cells $\left(1 \times 10^{4}\right)$ were seeded into the upper chamber coated with or without Matrigel (BD Bioscience, San Jose, CA, USA) and RPMI-1640 without FBS was added. Then, the chamber was placed into the cell culture plate containing RPMI-1640 supplemented with $10 \% \mathrm{FBS}$ and incubated at $37^{\circ} \mathrm{C}$ for $24 \mathrm{~h}$. Subsequently, the cells inside the upper chamber were carefully removed with cotton swabs. Migrated and invaded cells were fixed with $1 \%$ paraformaldehyde for $10 \mathrm{~min}$ and subsequently stained with hematoxylin for $5 \mathrm{~min}$. The migratory and invasive cells were finally counted in 10 independent vision and counted under a Leica TCS SP5 confocal microscope (Leica Microsystems, Wetzlar, Germany).

In vivo metastasis assay. Four- to six-week-old male BALB/c nude mice (Centre of Laboratory Animals, The Medical College of Xi'an Jiaotong University, Xi'an, China) were randomized into two groups $(n=5)$, and either the LV-TRIM14 or TRIM14-shRNA transfected cells $\left(1 \times 10^{6}\right)$ were injected into the tail veins for the establishments of pulmonary metastatic model. The mice were sacrificed 3 weeks post injection and examined microscopically by H\&E staining for the development of lung metastatic foci. Animals were housed in cages under standard conditions. All in vivo protocols were approved by the Institutional Animal Care and Use Committee of Xi'an Jiaotong University.

Immunohistochemical(IHC) staining. Paraformaldehyde-fixed paraffin GC tissue sections were used for IHC staining. TRIM14 primary antibodies (dilution 1:300; cat. no. ab85374; Abcam, Cambridge, MA, USA) were diluted in PBS to 1:100 and incubated at $4^{\circ} \mathrm{C}$ overnight. Sections were then incubated with biotinylated secondary antibodies (dilution 1:1,000; ZSGB-BIO, Beijing, China). Complexes were detected by HRP-streptavidin conjugates (ZSGB-BIO) and visualized with DAB (ZSGB-BIO).

Statistical analysis. All data are presented as mean \pm standard deviation (SD) and were analyzed using GraphPad Prism software version 5.0 (GraphPad Software, Inc., La Jolla, CA,
USA). Statistical analysis was calculated by a Chi-squared test, Student's t-test, ANOVA, Pearson correlation analysis, Kaplan-Meier method and log-rank test. P-value $<0.05$ was considered to indicate a statistically significant result. Each experiment was repeated three times.

\section{Results}

TRIM14 is upregulated in GC tissues and cell lines and is correlated with the survival of GC patients. To determine the expression level of TRIM14 in GC, we performed IHC staining to confirm TRIM14 expression and found that the IHC scores of TRIM14 in GC tissues were obviously increased compared to the scores noted in the normal tissues $(\mathrm{P}<0.05$, Fig. 1A). Kaplan-Meier survival cure demonstrated that an increased TRIM14 in GC patients was indicative of a shorter overall survival (OS) in GC patients $(\mathrm{P}=0.0002$, Fig. 1B). Subsequently, we randomly selected $40 \mathrm{GC}$ tissues and paired adjacent normal tissues to perform western blot analysis to validate these findings. Our data showed that the expression of TRIM14 protein was significantly higher in GC tissues than that in adjacent non-tumor tissues $(\mathrm{P}<0.05$; Fig. 2A). Furthermore, we analyzed TRIM14 expression in GC cell lines and the normal immortalized gastric epithelium cell line GES-1. The results showed that TRIM14 was increased in GC cell lines when compared with the level in GES-1 cells $(\mathrm{P}<0.05$; Fig. $2 \mathrm{~B})$. These results suggest that TRIM14 is involved in GC progression.

Clinical significance of TRIM14 in GC patients. To investigate the clinical role of TRIM14, we analyzed the relevance between TRIM14 and the clinicopathological features and prognosis of GC patients. We determine the mean value of expression as the cut-off and high TRIM14 was significantly associated with lymph node metastasis and advanced TNM stage $(\mathrm{P}=0.007$ and 0.02, respectively, Table I). These results suggest that TRIM14 is involved in the process of metastasis of GC.

Knockdown of TRIM14 attenuates the gastric cancer cell migration and invasion. Since our clinical data revealed the 
A
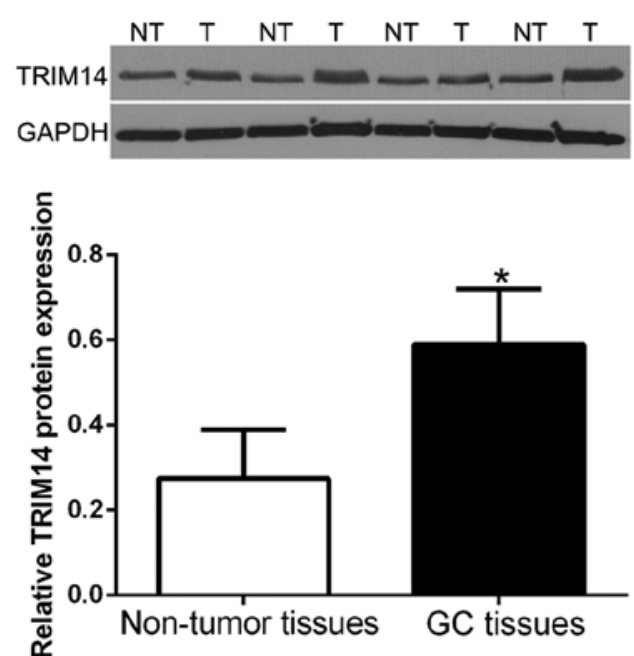

B
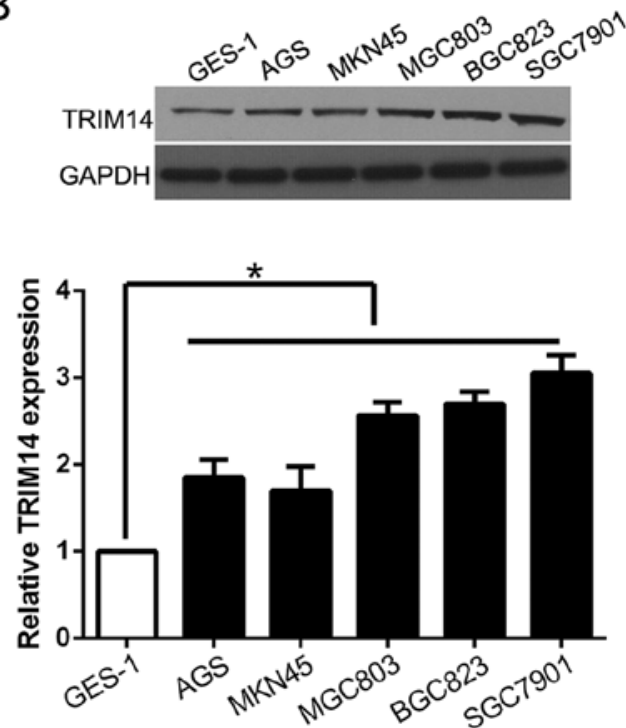

Figure 2. TRIM14 is frequently overexpressed in GC tissues and cell lines. (A) Representative western blot analysis of TRIM14 expression in GC (T) and corresponding matched adjacent nontumor tissues (NT) is shown; " $\mathrm{P}<0.05$ by t-test. (B) Comparison of the differences in the expression level of TRIM14 protein between GC cell lines with different metastatic potential and the immortalized gastric epithelium cell line GES-1. $\mathrm{n}=$ three repeats with similar results; "P $<0.05$ by ANOVA. TRIM14, tripartite motif-containing 14; GC, gastric cancer.

Table I. Association between TRIM14 expression and clinicopathological features of the GC patients ( $\mathrm{n}=117)$.

\begin{tabular}{|c|c|c|c|c|}
\hline \multirow[b]{2}{*}{ Clinical parameters } & \multirow[b]{2}{*}{ Cases (n) } & \multicolumn{2}{|c|}{ Expression level } & \multirow[b]{2}{*}{ Pvalue } \\
\hline & & TRIM14 $^{\text {high }}(\mathrm{n}=59)$ & TRIM14 $^{\text {low }}(\mathrm{n}=58)$ & \\
\hline Age (years) & & & & 0.629 \\
\hline$<60$ & 49 & 26 & 23 & \\
\hline$\geq 60$ & 68 & 33 & 35 & \\
\hline Sex & & & & 0.516 \\
\hline Male & 76 & 40 & 36 & \\
\hline Female & 41 & 19 & 22 & \\
\hline Tumor size $(\mathrm{cm})$ & & & & 0.227 \\
\hline$<$ & 94 & 50 & 44 & \\
\hline$\geq 5$ & 23 & 9 & 14 & \\
\hline Histological type & & & & 0.961 \\
\hline Intestinal & 91 & 46 & 45 & \\
\hline Diffuse & 26 & 13 & 13 & \\
\hline TNM stage & & & & $0.020^{\mathrm{a}}$ \\
\hline $\mathrm{I}+\mathrm{II}$ & 33 & 11 & 22 & \\
\hline III+IV & 84 & 48 & 36 & \\
\hline Lymph node metastasis & & & & $0.007^{\mathrm{a}}$ \\
\hline Present & 82 & 48 & 34 & \\
\hline Absent & 35 & 11 & 24 & \\
\hline
\end{tabular}

${ }^{a} \mathrm{P}<0.05$, significant difference.

correlation between TRIM14 and metastatic features of GC, we performed Matrigel uncoated and coated assays to determine the migration and invasion capacity of GC. MKN45 cells with low TRIM14 level and SGC7901 cells with high TRIM14 level were used for gain- and loss-of-function experiments, respectively ( $\mathrm{P}<0.05$, Fig. 3A and $\mathrm{C})$. Transwell assays showed that TRIM14 overexpression significantly promoted the migration and invasion of MKN45 cells $(\mathrm{P}<0.05$, Fig. 3B), while TRIM14 knockdown significantly inhibited migration and invasion of SGC7901 cells ( $\mathrm{P}<0.05$, Fig. 3D). Furthermore, 
A

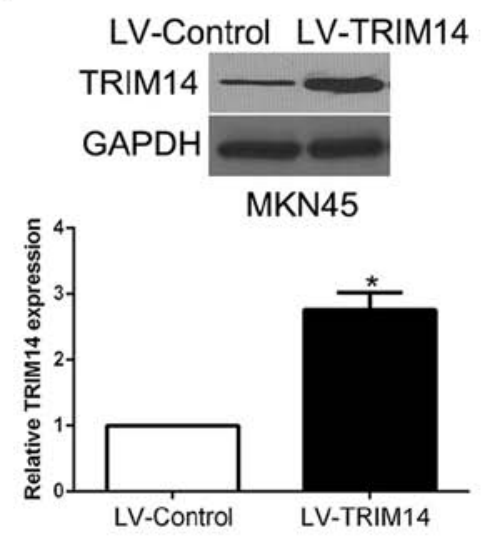

C

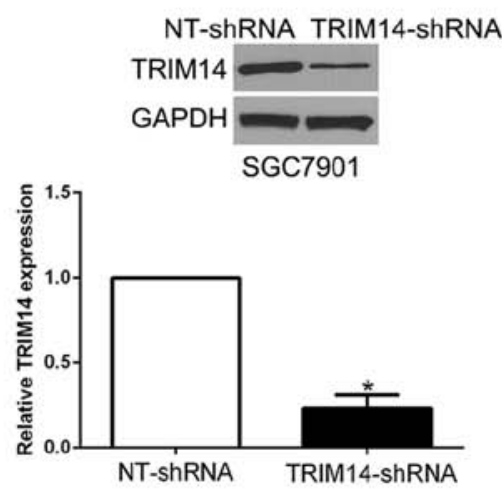

B
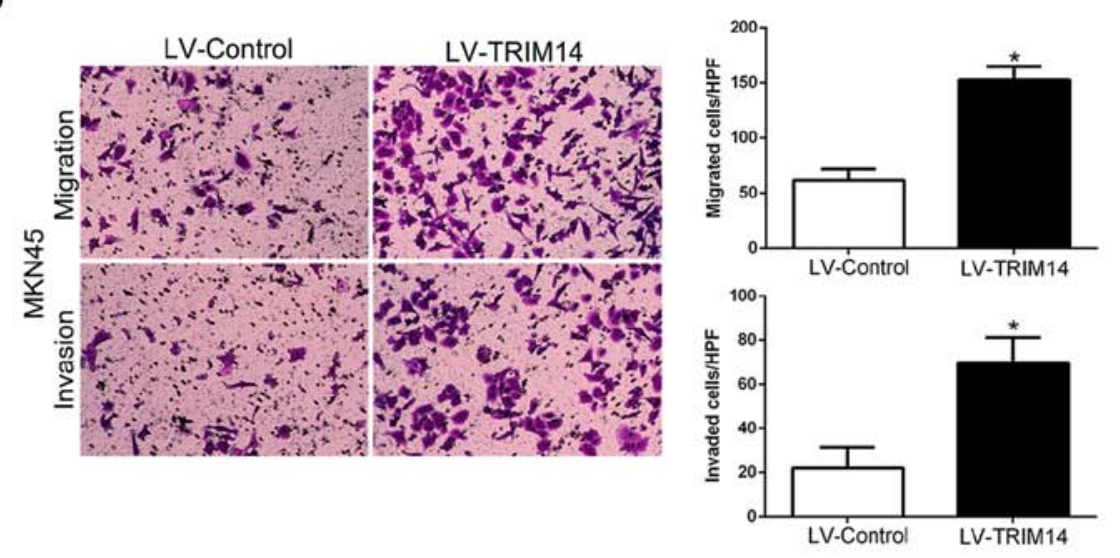

D

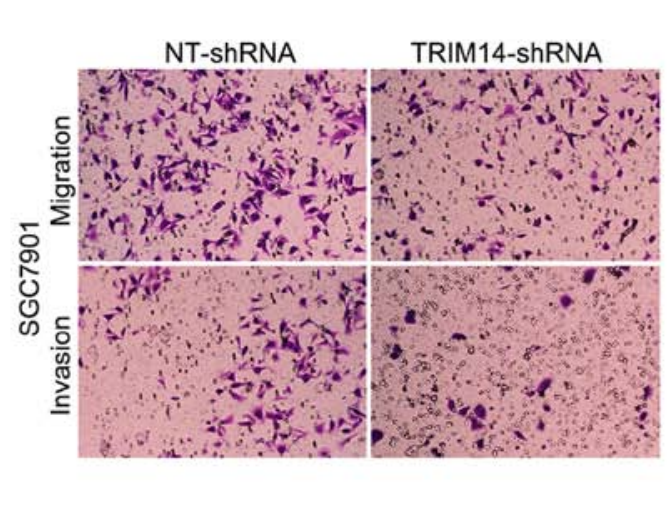

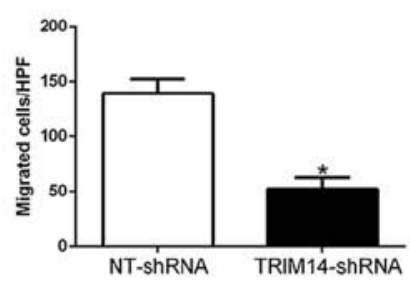

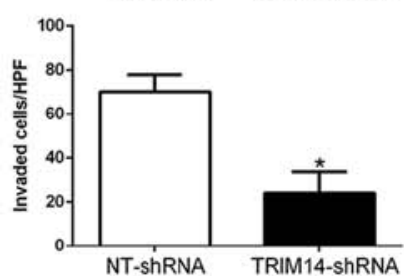

E

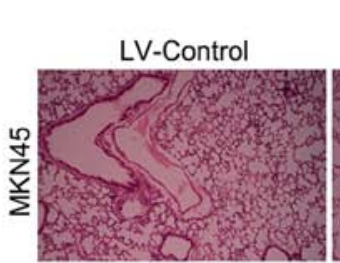

NT-shRNA

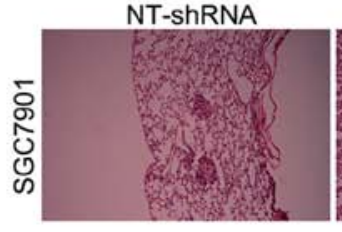

LV-TRIM14

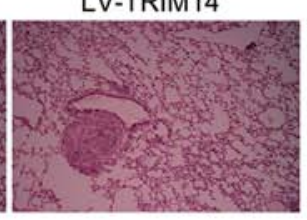

TRIM14-shRNA
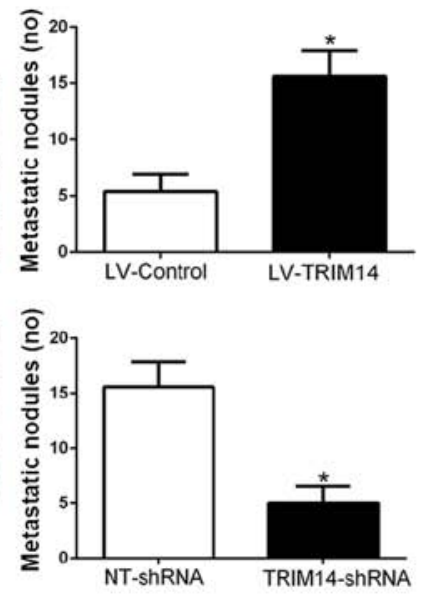

Figure 3. TRIM14 promotes GC cell migration and invasion in vitro and in vivo. (A) MKN45 cells that were transfected with the corresponding TRIM14 overexpression vectors (LV-TRIM14 and LV-Control) were subjected to western blotting for TRIM14. (B) Cell migration and invasion as measured by Transwell assays were promoted by overexpression of TRIM14 in MKN45 cells. (C) SGC7901 cells that were transfected with TRIM14-shRNA and negative control (NT-shRNA) were subjected to western blotting for TRIM14. (D) Cell migration and invasion as measured by Transwell assays were inhibited by knockdown of TRIM14 in SGC7901 cells. (E) Representative H\&E staining of lung metastasis of MKN45 or SGC7901 cells transfected with the respective TRIM14 vector. $n=$ six independent experiments. "P<0.05. TRIM14, tripartite motif-containing 14; GC, gastric cancer; HPF, high-power field.

to confirm the role of TRIM14 in vivo, we used tail vain injection to construct a lung metastasis model. Our results showed that TRIM14 overexpression significantly increased the number of lung metastatic nodules derived from MKN45 cells whereas TRIM14 knockdown validly reduced the lung metastasis $(\mathrm{P}<0.05$, Fig. 3E). Therefore, TRIM14 displays a critical role in the migration and invasion of GC cells in vitro and in vivo. 
A

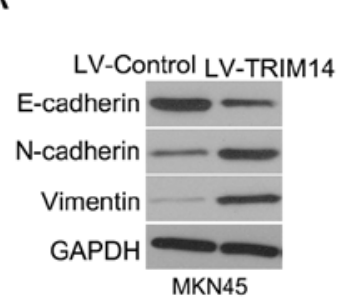

C

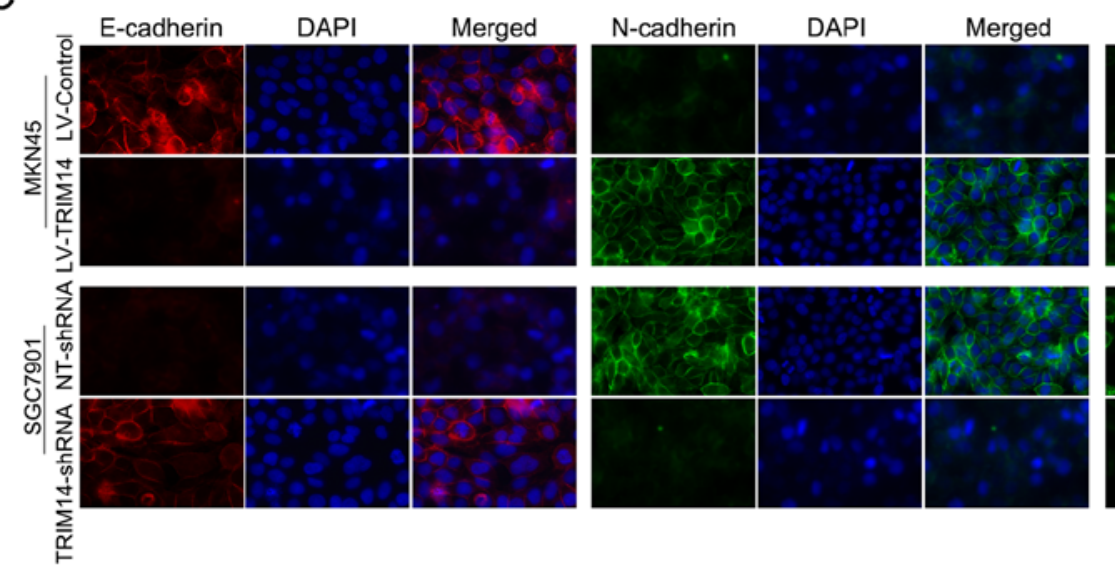

B
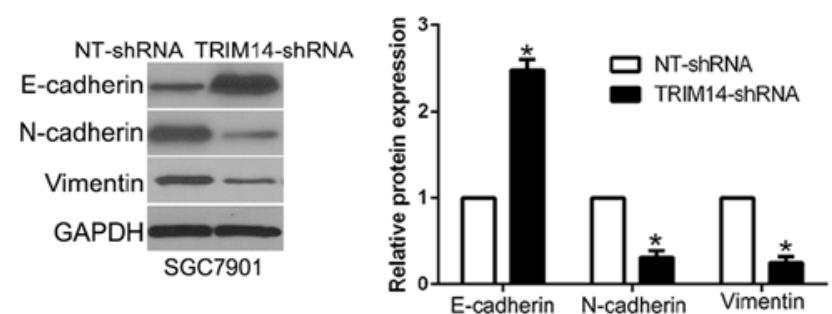

D
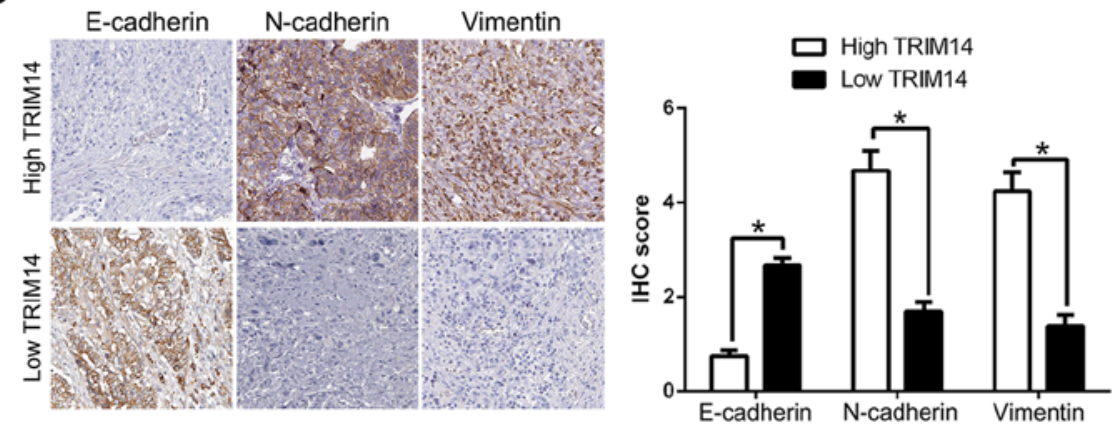

Figure 4. TRIM14 promotes epithelial-to-mesenchymal transition in GC cells. The overexpression of TRIM14 in MKN45 cells reduced the expression of the epithelial cell marker E-cadherin and increased the expression of mesenchymal cell markers N-cadherin and vimentin as determined by (A) western blot analysis and (C) immunofluorescence (IF). In contrast, TRIM14 knockdown increased E-cadherin expression and reduced N-cadherin and vimentin expression as determined by (B) western blot analysis and (C) IF. (D) Immunohistochemical analysis of E-cadherin, N-cadherin and vimentin in GC samples. In cases of high TRIM14 expression ( $\mathrm{n}=59$ ); there was no detectable E-cadherin (upper left panel) and strong N-cadherin, vimentin protein (upper middle and right panels) expression in the tissue section. In contrast, in the case of low TRIM14 expression ( $\mathrm{n}=58$ ), there was strong E-cadherin (lower left panel) and no detectable $\mathrm{N}$-cadherin and vimentin protein (lower middle and right panels) expression. Values are depicted as mean \pm SEM; ${ }^{*} \mathrm{P}<0.05$ by t-test. TRIM14, tripartite motif-containing 14; GC, gastric cancer. TRIM14 overexpression and control vectors (LV-TRIM14 and LV-Control) and TRIM14 knockdown and negative control (TRIM14-shRNA and NT-shRNA).

TRIM14 promotes EMT in GC. EMT plays a critical role in tumor invasion and metastasis (18). To determine the effect of TRIM14 on EMT, western blot analysis showed that TRIM14 overexpression significantly decreased the level of epithelial marker E-cadherin, while it increased the levels of mesenchymal markers $\mathrm{N}$-cadherin and vimentin $(\mathrm{P}<0.05$, Fig. 4A). In contrary, TRIM14 knockdown showed the opposite effects $(\mathrm{P}<0.05$, Fig. 4B). Moreover, immunofluorescence (IF) confirmed the similar function of TRIM14 on the process of EMT (Fig. 4C). In addition, we demonstrated that E-cadherin was lower in the GC tissues with high TRIM14 expression while vimentin was remarkably higher in GC tissues with high TRIM14 than that in the low TRIM14 GC tissue group ( $<<0.05$, Fig. 4D). Taken together, we verified that TRIM14 is an activator of the EMT process in GC.

AKT phosphorylation mediates the biological effects of TRIM14 in GC cells. Previous research confirmed that AKT signaling plays an important role in GC metastasis (19). Moreover, among the TRIM family, TRIM29 stimulates the AKT pathway in human cancers (20). Thus, we aimed to ascertain whether TRIM14 regulates the AKT pathway in GC. Notably, TRIM14 overexpression promoted p-AKT, p-mTOR and p-P70S6K expression while TRIM14 knockdown showed the opposite effects $(\mathrm{P}<0.05$, Fig. 5A). To ascertain whether the AKT pathway mediates the effects of TRIM14, we used AKT inhibitor MK2206 to inhibit AKT 
A
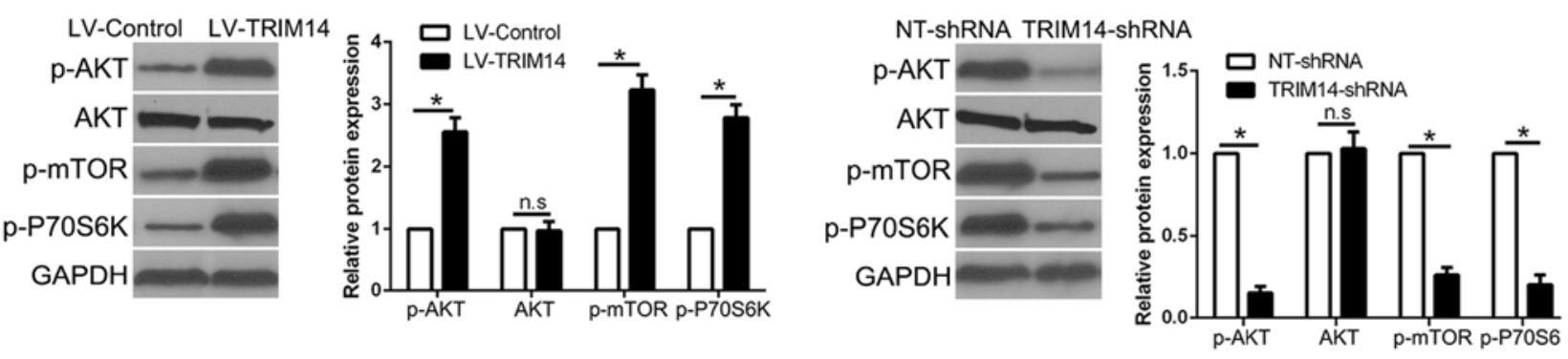

B
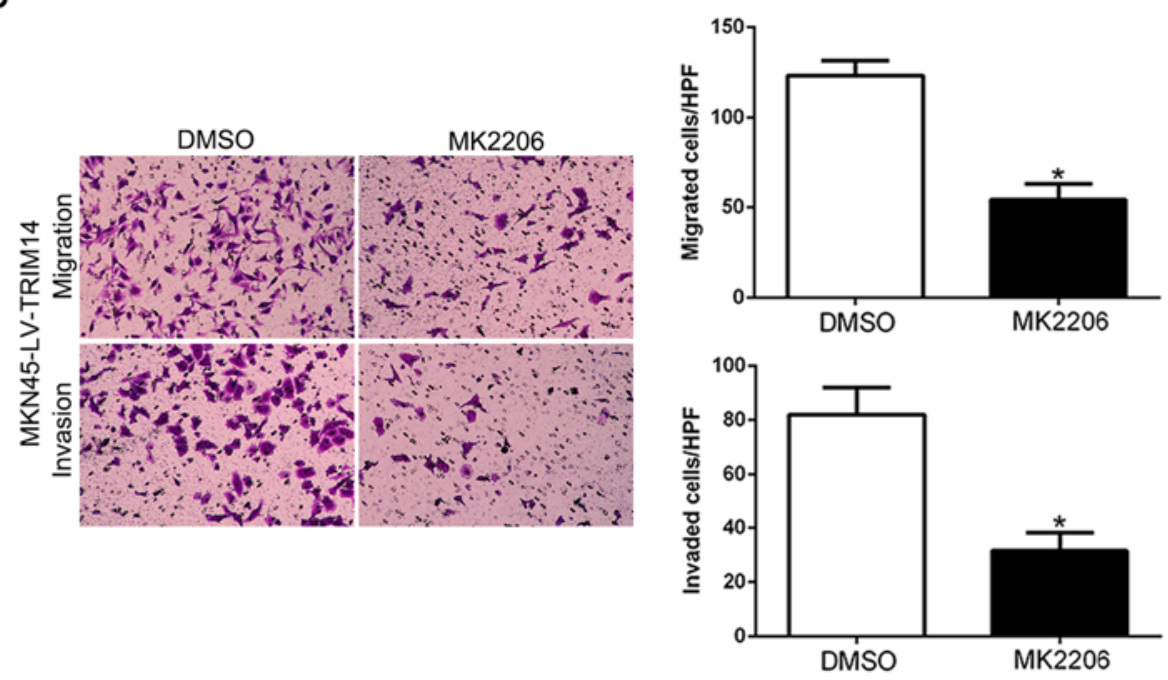

C
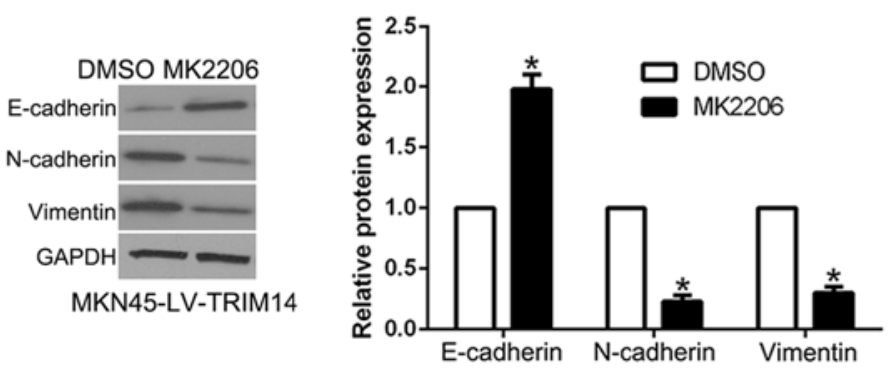

Figure 5. TRIM14 regulates GC cell migration and invasion via activation of the AKT signaling pathway. (A) Western blot analysis of the indicated AKT signaling pathway proteins in the cells after TRIM14 overexpression (LV-TRIM14 and LV-Control) or knockdown (TRIM14-shRNA and NT-shRNA) in GC cells. (B) MKN45/TRIM14-overexpressing cells were treated with or without AKT inhibitor MK2206, for 24 h, and cell migration and invasion abilities were determined by Transwell assays, respectively. (C) Western blot analysis of the EMT-associated proteins after treatment with the AKT inhibitor. ${ }^{*} \mathrm{P}<0.05$; n.s., not significant. TRIM14, tripartite motif-containing 14; GC, gastric cancer.

phosphorylation in TRIM14-overexpressing GC cells. The results showed that $\mathrm{AKT}$ inhibition reversed the promotive effects of TRIM14 in regards to cell migration and invasion $(\mathrm{P}<0.05$, Fig. 5B). Similarly, AKT inhibition abolished the effects of TRIM14 on EMT progression ( $\mathrm{P}<0.05$, Fig. $5 \mathrm{C})$. Collectively, these data suggest that the AKT pathway at least partially mediated the effects of TRIM14 on migration and invasion of GC.

TRIM14 expression is regulated by miR-195-5p. Previous research has confirmed that aberrant miRNA expression contributes to GC progression and regulates protein expression by binding to its 3'UTR (21). To elucidate the mechanisms involved in the upregulation of TRIM14, we searched the
TargetScan to predict whether miR-195-5p could bind to the 3'UTR of TRIM14 (Fig. 6C). We performed qRT-PCR to confirm that TRIM14 mRNA expression was higher in GC tissues than those in adjacent non-tumor tissues $(\mathrm{P}<0.05$, Fig. 6A). Notably, an inverse correlation between TRIM14 mRNA and miR-195-5p was confirmed in GC tissues ( $r=-0.864$, $\mathrm{P}<0.05$, Fig. 6B, left graph). Furthermore, miR-195-5p expression in the high TRIM14 group tissues was prominently lower than that in the GC tissues with low TRIM14 expression $(\mathrm{P}<0.05$, Fig. 6B, right histogram). Luciferase reporter assays confirmed that miR-195-5p overexpression prominently reduced while miR-195-5p knockdown increased the luciferase activity of cells with wt 3'UTR of TRIM14 $(\mathrm{P}<0.05$, Fig. 6D). However, the activity had no change in $\mathrm{mt}$ 3'UTR 
A

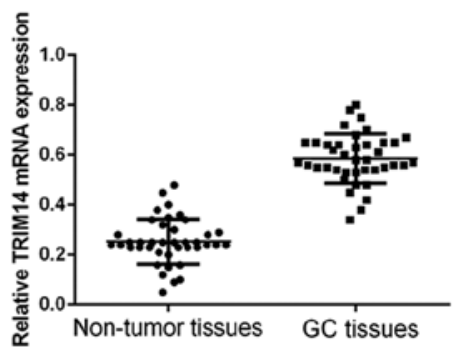

B

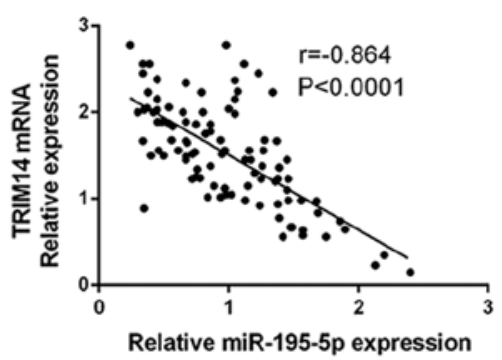

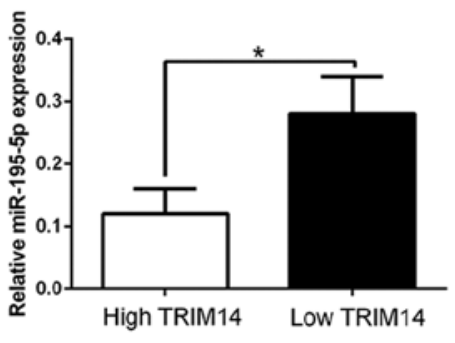

C

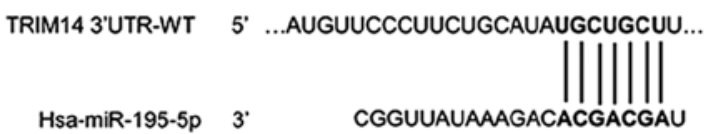

TRIM14 3'UTR-MT $\quad 5$ ' ...AUGUUCCCUUCUGCAUAAAGGAAGU...

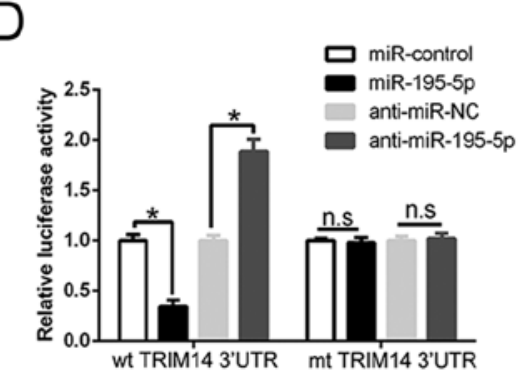

$E$
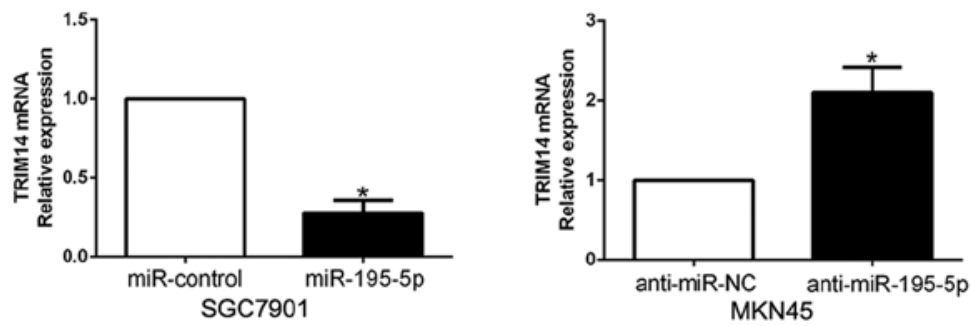

$\mathrm{F}$

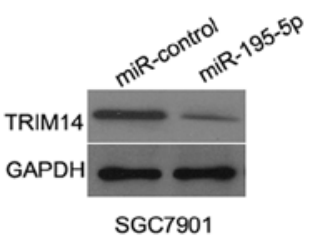

SGC7901

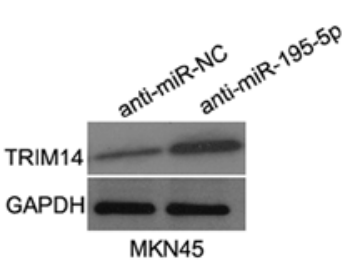

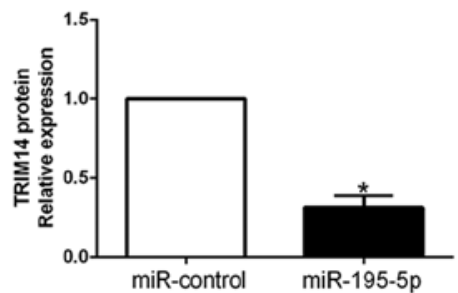

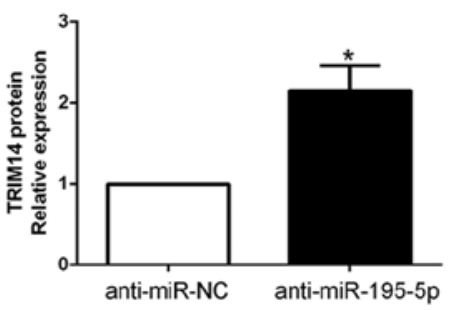

G

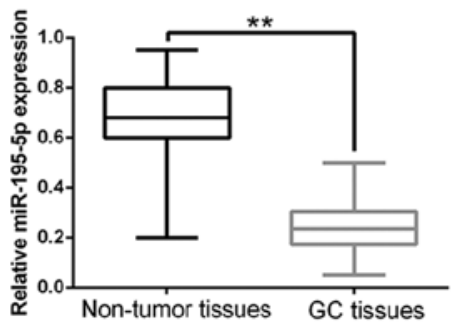

Figure 6. TRIM14 is identified as a direct target of miR-195-5p in GC. (A) Relative TRIM14 mRNA expression levels in tumor tissues (T) and matched adjacent non-tumor tissues (NT) were determined by qRT-PCR. (B) A significant inverse correlation between the mRNA levels of TRIM14 and miR-195-5p was observed in GC tissues. The expression of miR-195-5p in TRIM14 high-expressing tumors was significantly lower than that in TRIM14 low-expressing tumors. (C) miR-195-5p and its putative binding sequence in the 3'UTR of TRIM14. The mutant binding site was generated in the complementary site for the seed region of miR-195-5p. (D) miR-195-5p significantly suppressed the luciferase activity that carried the wild-type (wt) but not mutant (mt) 3 'UTR of TRIM14. Anti-miR-195-5p led to a notable increase in the luciferase activity of wt 3'-UTR of TRIM14. (E) qRT-PCR analysis of TRIM14 mRNA expression in SGC7901 cells transfected with miR-195-5p or miR-control vector and MKN45 cells with anti-miR-195-5p or anti-miR-NC vector transfection. (F) Overexpression of miR-195-5p reduced the expression of TRIM14 protein in SGC7901 cells and knockdown of miR-195-5p increased the level of TRIM14 protein in MKN45 cells. (G) The expression level of miR-195-5p in GC tissues and adjacent non-tumor tissues. $\mathrm{n}=$ six repeats with similar results. ${ }^{*} \mathrm{P}<0.05$, ${ }^{* *} \mathrm{P}<0.01$. TRIM14, tripartite motif-containing 14; GC, gastric cancer. 
B

A

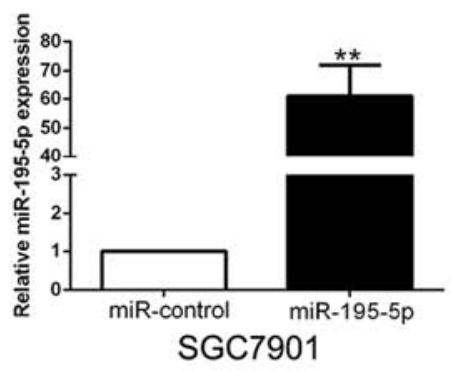

C

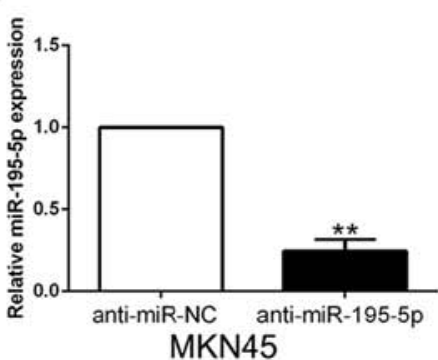

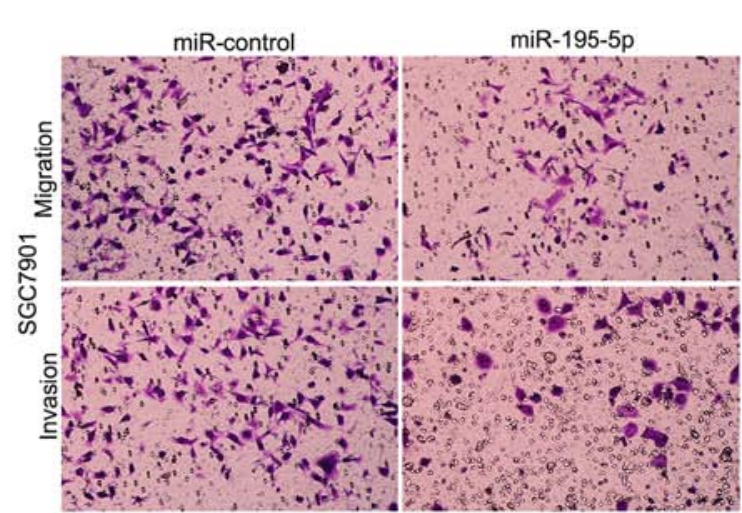
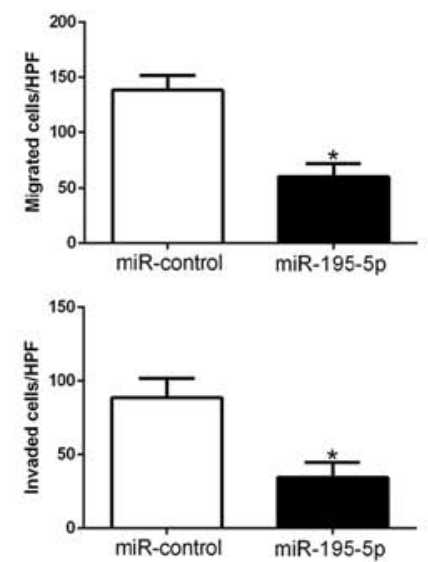

D

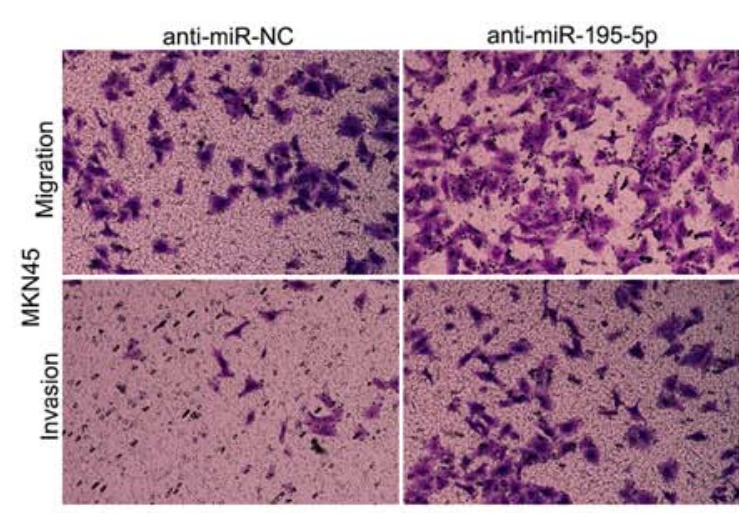

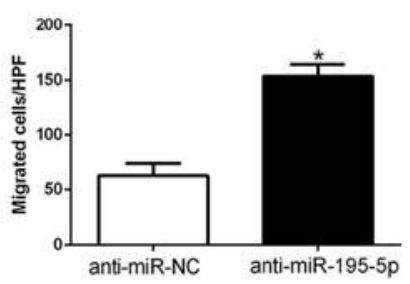

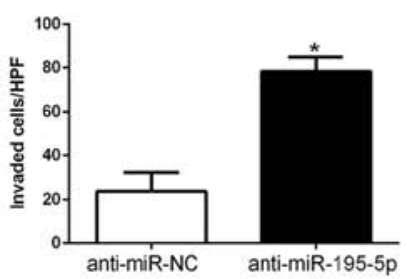

Figure 7. miR-195-5p inhibits GC cell migration and invasion in vitro. (A) SGC7901 cells transfected with miR-195-5p or miR-control vector were subjected to qRT-PCR for miR-195-5p. (B) Cell migration and invasion as measured by Transwell assays were inhibited by overexpression of miR-195-5p in SGC7901 cells. (C) MKN45 cells that were transfected with miR-195-5p inhibitors (anti-miR-195-5p) and negative control (anti-miR-NC) were subjected to qRT-PCR for miR-195-5p. (D) Cell migration and invasion abilities as measured by Transwell assays were increased by knockdown of miR-195-5p in MKN45 cells. $n=$ six independent experiments. ${ }^{*} \mathrm{P}<0.05,{ }^{* *} \mathrm{P}<0.01$. TRIM14, tripartite motif-containing 14; GC, gastric cancer.

of TRIM14. Moreover, miR-195-5p overexpression significantly inhibited while miR-195-5p knockdown promoted TRIM14 mRNA and protein in GC cells $(\mathrm{P}<0.05$, respectively, Fig. 6E and F). In GC tissues, we demonstrated that miR-195-5p was downregulated compared to that noted in the adjacent non-tumor tissues $(\mathrm{P}<0.05$, Fig. 6G). Taken together, we first disclose that miR-195-5p regulates TRIM14 expression in GC tissues.

miR-195-5p inhibits the migration and invasion of GC cells. To confirm the biological function of miR-195-5p in GC tissues, we used overexpression or knockdown vectors to perform gain- and loss-of-function experiment. The Transwell assays showed that miR-195-5p overexpression significantly inhibited the migration and invasion of SGC7901 cells $(\mathrm{P}<0.05$, Fig. 7A and B). Furthermore, miR-195-5p knockdown significantly facilitated the migration and invasion of MKN45 cells ( $\mathrm{P}<0.05$, Fig. 7C and D). Thus, miR-195-5p functions as a tumor suppressor in the migration and invasion of GC cells.

TRIM14 mediates the effects of miR-195-5p in GC cells. To clarify whether TRIM14 is a biological mediator of
miR-195-5p, we perform a rescue experiment in which TRIM14 was restored in miR-195-5p-overexpressing SGC7901 cells $(\mathrm{P}<0.05$, Fig. 8A). TRIM14 restoration reversed the inhibitory effect of miR-195-5p on the migration and invasion of SGC7901 cells $(\mathrm{P}<0.05$, Fig. 8B). Moreover, TRIM14 restoration increased the levels of $\mathrm{p}$-AKT, N-cadherin and vimentin and reduced E-cadherin expression in the miR-195-5p-overexpressing SGC7901 cells ( $\mathrm{P}<0.05$, Fig. 8E). However, TRIM14 knockdown significantly inhibited the migration, invasion and EMT progression of miR-195-5p-suppressed MKN45 cells $(\mathrm{P}<0.05$, Fig. 8C, D and F). These results suggest that TRIM14 is a downstream mediator of miR-195-5p in GC.

\section{Discussion}

The TRIM14 superfamily of proteins are important regulators of cellular physiological and pathological processes including cell proliferation, apoptosis, inflammation, immunity and metastasis $(8,22,23)$. Recently, an increasing body of evidence has confirmed that TRIM14 is involved in cancer (24). TRIM14 was found to regulate cell proliferation and invasion in osteosarcoma via promotion of AKT signaling (8). 
B

A

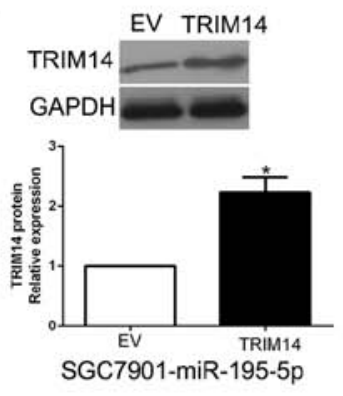

C
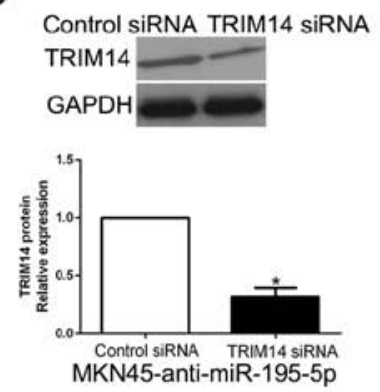

D
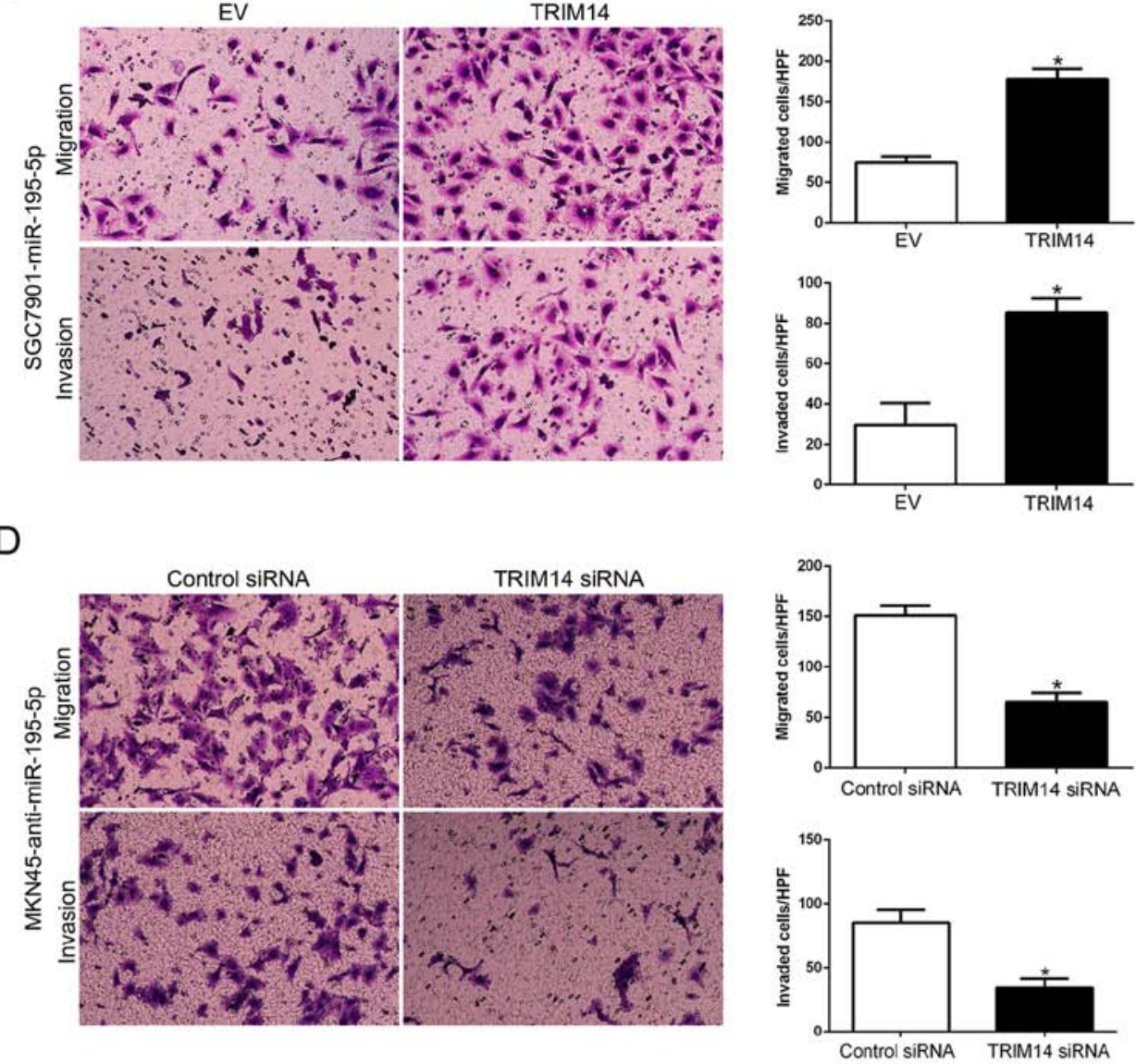

E
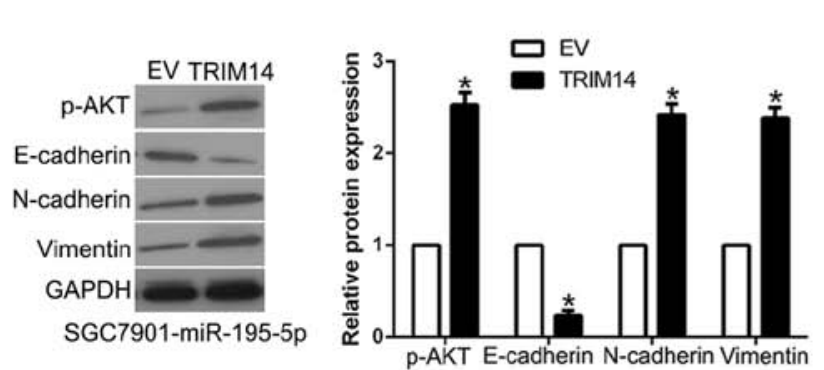

F
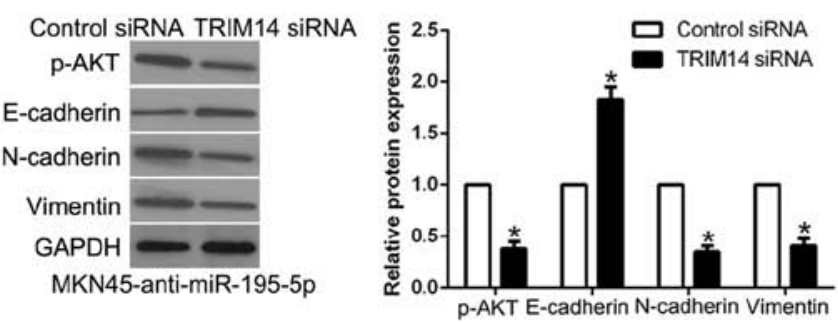

Figure 8. Alterations in the expression of TRIM14 partially abolish miR-195-5p-mediated GC cell migration,invasion and EMT progression.(A) miR-195-5p-overexpressing SGC7901 cells that were transfected with EV or TRIM14 expression plasmid were subjected to western blot analysis for TRIM14. (B) Cell migration and invasion of the miR-195-5p-overexpressing SGC7901 cells was increased by TRIM14 overexpression. (C) miR-195-5p-silenced MKN45 cells that were transfected with scrambled siRNA or TRIM14-siRNA were subjected to western blot analysis for TRIM14. (D) TRIM14 knockdown abrogated the effects of miR-195-5p knockdown on MKN45 cells. (E) Western blot analysis of EMT marker proteins in SGC7901 cells stably expressing miR-195-5p transduced with TRIM14 or control vector. (F) Western blot analysis of indicated proteins in MKN45 cells stably expressing miR-195-5p inhibitor transfected with TRIM14 siRNA or control siRNA. $\mathrm{n}=$ six independent experiments. ${ }^{*} \mathrm{P}<0.05$. EV, empty vector; TRIM14, tripartite motif-containing 14 ; GC, gastric cancer.

Moreover, a bispecific antibody against TRIM14 suppressed osteosarcoma aggressiveness through regulation of the $\mathrm{NF}-\kappa \mathrm{B}$ signaling pathway (25). Here, we reported for the first time that TRIM14 mRNA and protein were both increased in GC tissues and cell lines. Our clinical data showed that high TRIM14 was significantly correlated with advanced TNM stage and lymph node metastasis of GC patients. Moreover, TRIM14 was found to be an important prognostic marker for 5-year OS in GC patients. These results showed that TRIM14 play a critical role in aggressive clinical characteristics and unfavorable prognosis, and the development and progression of GC.
To confirm the biological function of TRIM14 in GC, we performed gain- and loss-of-function experiment in vitro and in vivo to confirm that TRIM14 promotes migration and invasion of GC cells by regulating EMT phenotype progression. EMT is critical for GC metastasis. We used western blot analysis and immunofluorescence to confirm that TRIM14 regulates the EMT process. Moreover, in GC tissues, TRIM14 was also significantly associated with the EMT process. To explore the underlying TRIM14-induced migration, invasion and EMT process in GC cells, we demonstrated that AKT signaling was activated and inhibition of AKT signaling reversed the TRIM14-induced migration, invasion and EMT 
progression. This is consistent with previous results that TRIM14 activates the AKT signaling pathway-mediated cascade which is involved in various processes.

Previous studies have reported that abnormal expression of miRNAs has been verified to be vital regulators in the initiation and progression of human cancers $(26,27)$. We searched bioinformation database and showed that miR-195-5p could interact with TRIM14 3'UTR. Luciferase reporter assays confirmed that miR-195-5p could directly bind to the 3'UTR of TRIM14. Gainand loss-of-function experiments confirmed that miR-195-5p negatively regulated the expression of TRIM14 mRNA and protein. In GC tissues, miR-195-5p showed an inverse correlation with TRIM14 expression. Moreover, alteration of miR-195-5p expression resulted in an inhibitory effect on migration and invasion of GC cells. Through rescue experiments, we demonstrated that alteration of TRIM14 abolished the effects of miR-195-5p on migration, invasion and EMT progression in GC cells. These results suggest that TRIM14 is a downstream target and mediator of miR-195-5p in GC.

In conclusion, we demonstrated that TRIM14 is increased in GC tissues and cell lines. Its high expression is associated with malignant clinical features and unfavorable prognosis. Gainand loss-of-functional experiments confirmed that TRIM14 promotes migration, invasion and EMT progression by activating AKT signaling. Additionally, we determined that miR-195-5p regulates TRIM14 expression and inhibits migration and invasion of GC cells. Moreover, alteration of TRIM14 expression abolished the effects of miR-195-5p on migration, invasion and the EMT process in GC. These findings provide an improved understanding of GC progression and support the potential role of TRIM14 as an attractive therapeutic target for GC treatment.

\section{Acknowledgements}

Not applicable.

\section{Funding}

This study was supported by the Fundamental Research Funds for the Central Universities (no. 1191329732).

\section{Availability of data and materials}

The datasets used during the present study are available from the corresponding author upon reasonable request.

\section{Authors' contributions}

FW, JY, QZ and WW performed all the experiments and drafted the manuscript. LR and FW participated in the study design, data analysis and interpretation of results. All authors read and approved the final manuscript and agree to be accountable for all aspects of the research in ensuring that the accuracy or integrity of any part of the work are appropriately investigated and resolved.

\section{Ethics approval and consent to participate}

The present study was approved by the Ethics Committee of The First Affiliated Hospital of Xi'an Jiaotong University and written informed consent was obtained from all enrolled patients. All in vivo protocols were approved by the Institutional Animal Care and Use Committee of Xi'an Jiaotong University.

\section{Patient consent for publication}

Not applicable.

\section{Competing interests}

The authors declare that they have no competing interests.

\section{References}

1. Siegel R, Ma J, Zou Z and Jemal A: Cancer statistics, 2014. CA Cancer J Clin 64: 9-29, 2014.

2. Torre LA, Bray F, Siegel RL, Ferlay J, Lortet-Tieulent J and Jemal A: Global cancer statistics, 2012. CA Cancer J Clin 65: 87-108, 2015.

3. Zong L, Abe M, Seto Y and Ji J: The challenge of screening for early gastric cancer in China. Lancet 388: 2606, 2016.

4. Cervantes A, RodaD, Tarazona N, Roselló S and Pérez-Fidalgo JA: Current questions for the treatment of advanced gastric cancer. Cancer Treat Rev 39: 60-67, 2013.

5. Glockzin G and Piso P: Current status and future directions in gastric cancer with peritoneal dissemination. Surg Oncol Clin N Am 21: 625-633, 2012.

6. Meroni G and Diez-Roux G: TRIM/RBCC, a novel class of 'single protein RING finger' E3 ubiquitin ligases. Bioessays 27: 1147-1157, 2005.

7. Wang $\mathrm{X}$, Guo H, Yao B and Helms J: miR-15b inhibits cancer-initiating cell phenotypes and chemoresistance of cisplatin by targeting TRIM14 in oral tongue squamous cell cancer. Oncol Rep 37: 2720-2726, 2017.

8. Xu G, Guo Y, Xu D, Wang Y, Shen Y, Wang F, Lv Y, Song F, Jiang D, Zhang Y, et al: TRIM14 regulates cell proliferation and invasion in osteosarcoma via promotion of the AKT signaling pathway. Sci Rep 7: 42411, 2017.

9. Dong B and Zhang W: High levels of TRIM14 are associated with poor prognosis in hepatocellular carcinoma. Oncol Res Treat 41: 129-134, 2018

10. Su X, Wang J, Chen W, Li Z, Fu X and Yang A: Overexpression of TRIM14 promotes tongue squamous cell carcinoma aggressiveness by activating the NF- $\mathrm{KB}$ signaling pathway. Oncotarget 7 : 9939-9950, 2016.

11. Hu G, Pen W and Wang M: TRIM14 promotes breast cancer cell proliferation by inhibiting apoptosis. Oncol Res: Mar 21, 2018 (Epub ahead of print).

12. Hai J, Zhu CQ, Wang T, Organ SL, Shepherd FA and Tsao MS: TRIM14 is a putative tumor suppressor and regulator of innate immune response in non-small cell lung cancer. Sci Rep 7: 39692, 2017.

13. Wu Q, Xiang S, Ma J, Hui P, Wang T, Meng W, Shi M and Wang Y: Long non-coding RNA CASC15 regulates gastric cancer cell proliferation, migration and epithelial mesenchymal transition by targeting CDKN1A and ZEB1. Mol Oncol 12: 799-813, 2018

14. Liu Z, Dou C, Yao B, Xu M, Ding L, Wang Y, Jia Y, Li Q, Zhang $\mathrm{H}$, Tu K, et al: Methylation-mediated repression of microRNA-129-2 suppresses cell aggressiveness by inhibiting high mobility group box 1 in human hepatocellular carcinoma. Oncotarget 7: 36909-36923, 2016.

15. Chen Y, Liu J, Wang W, Xiang L, Wang J, Liu S, Zhou H and Guo Z: High expression of hnRNPA1 promotes cell invasion by inducing EMT in gastric cancer. Oncol Rep 39: 1693-1701, 2018.

16. Zhao J, Geng L, Duan G, Xu W, Cheng Y, Huang Z, Zhou Z and Gong S: REC8 inhibits EMT by downregulating EGR1 in gastric cancer cells. Oncol Rep 39: 1583-1590, 2018.

17. Livak KJ and Schmittgen TD: Analysis of relative gene expression data using real-time quantitative PCR and the 2(-Delta Delta C(T)) method. Methods 25: 402-408, 2001.

18. Ceausu AR, Ciolofan A, Cimpean AM, Magheti A, Mederle O and Raica M: The mesenchymal-epithelial and epithelial-mesenchymal cellular plasticity of liver metastases with digestive origin. Anticancer Res 38: 811-816, 2018. 
19. Chen D, Lin X, Zhang C, Liu Z, Chen Z, Li Z, Wang J, Li B, Hu Y, Dong B, et al: Dual PI3K/mTOR inhibitor BEZ235 as a promising therapeutic strategy against paclitaxel-resistant gastric cancer via targeting PI3K/Akt/mTOR pathway. Cell death Dis 9: 123, 2018.

20. Xu J, Li Z, Su Q, Zhao J and Ma J: TRIM29 promotes progression of thyroid carcinoma via activating P13K/AKT signaling pathway. Oncol Rep 37: 1555-1564, 2017.

21. Xiao C, Hong H, Yu H, Yuan J, Guo C, Cao H and Li W: MiR-340 affects gastric cancer cell proliferation, cycle, and apoptosis through regulating SOCS3/JAK-STAT signaling pathway. Immunopharmacol Immunotoxicol 40: 278-283, 2018

22. Tan P, He L, Cui J, Qian C, Cao X, Lin M, Zhu Q, Li Y, Xing C, Yu X, et al: Assembly of the WHIP-TRIM14-PPP6C mitochondrial complex promotes RIG-I-mediated antiviral signaling. Mol Cell 68: 293-307.e5, 2017.

23. Jia X, Zhou H, Wu C, Wu Q, Ma S, Wei C, Cao Y, Song J, Zhong H, Zhou Z and Wang J: The ubiquitin ligase RNF125 targets innate immune adaptor protein TRIM14 for ubiquitination and degradation. J Immunol 198: 4652-4658, 2017.

24. Wang T, Ren Y, Liu R, Ma J, Shi Y, Zhang L and Bu R: miR-195-5p suppresses the proliferation, migration, and invasion of oral squamous cell carcinoma by targeting TRIM14. Biomed Res Int 2017: 7378148, 2017.
25. Yu GH, Li AM, Li X, Yang Z and Peng H: Bispecific antibody suppresses osteosarcoma aggressiveness through regulation of NF-KB signaling pathway. Tumour Biol 39: 1010428317705572 , 2017.

26. Liu Z, Wang Y, Dou C, Sun L, Li Q, Wang L, Xu Q, Yang W, Liu Q and Tu K: MicroRNA-1468 promotes tumor progression by activating PPAR- $\gamma$-mediated AKT signaling in human hepatocellular carcinoma. J Exp Clin Cancer Res 37: 49, 2018.

27. Liu Z, Dou C, Yao B, Xu M, Ding L, Wang Y, Jia Y, Li Q, Zhang H, Tu K, et al: Ftx non coding RNA-derived miR-545 promotes cell proliferation by targeting RIG-I in hepatocellular carcinoma. Oncotarget 7: 25350-25365, 2016.

(i) (3) This work is licensed under a Creative Commons Attribution-NonCommercial-NoDerivatives 4.0 International (CC BY-NC-ND 4.0) License. 